\title{
Erythrocyte Flow Cytometric Analysis in Congenital Dyserythropoietic Anemia Type III-Evaluation of Eosin-5'-Maleimide, CD55, and CD59
}

Maria Liljeholm $^{1 *}$, Elisabeth Gronlund ${ }^{3}$, Irina Golovleva ${ }^{3}$, Herbert Sandström² and Anders Wahlin ${ }^{1}$

${ }^{1}$ Department of Radiation Sciences, Umea University, Sweden

${ }^{2}$ Department of Public Health and Clinical Medicine, Umea University, Sweden

${ }^{3}$ Department of Medical Biosciences, Umea University, Sweden

\begin{abstract}
Introduction: Flow cytometry with eosin- $5^{\prime}$-maleimide (EMA), anti-CD55 and anti-CD59 is commonly used when investigating non-autoimmune hemolytic anemias. Reduced fluorescence of EMA, typically detected in hereditary spherocytosis is also seen in congenital dyserythropoietic anemia type II (CDA II). Reduction of CD55 and CD59 characterizes paroxysmal nocturnal hemoglobinuria (PNH). We studied the flow cytometric profile of EMA, CD55 and CD59 on erythrocytes in congenital dyserythropoietic anemia type III (CDA III).

Methods: Erythrocytes from 16 CDA III positive individuals, 14 CDA III negative relatives and three normal controls per assay were studied with flow cytometry after EMA staining. Flow cytometry after anti-CD55 and antiCD59 was performed on erythrocytes from 12 CDA III positive and 7 CDA III negative relatives with one normal control per assay.

Results: CDA III - erythrocytes exhibited marginally stronger fluorescence after EMA-staining than normal controls. Correlation between EMA fluorescence and erythrocyte volume was confirmed. CDA III subjects did not differ from normal controls concerning CD55 and CD59.

Conclusion: The results of the present study indicate no abnormality of the erythrocyte membrane in CDA III and show that standard flow cytometry cannot be used to discriminate between CDA III and normal controls.
\end{abstract}

Keywords: Congenital dyserythropoietic anemia type III; Flow cytometry; Eosin-5' -maleimide; CD55; CD59

\section{Introduction}

Flow cytometry with recording of mean cellular fluorescence (MCF) after eosin-5'-maleimide (EMA) staining is a valuable tool in the work-up of patients with direct antiglobulin test (DAT) negative anemia [1,2]. EMA binds predominantly to band 3, but also to CD47 and Rhesus (Rh)-related glycoproteins, together leading to the markedly reduced EMA binding in hereditary spherocytosis [3,4]. EMA binding appears to be normal in most common anemias such as DAT positive hemolytic anemia and iron deficiency as well as in hemoglobinopathies such as thalassemia [5]. Normal MCF after EMA staining has also been reported in anemias due to enzymopathies such as glucose- 6 phosphate dehydrogenase deficiency and pyruvate kinase deficiency [6]. Reduced binding of monoclonal antibodies against the glycosylphosphatidylinositol anchor proteins CD55 and CD59 to erythrocytes and myeloid cells forms the basis of the flow cytometric diagnosis of paroxysmal nocturnal hemoglobinuria (PNH) [7].

Congenital dyserythropoietic anemias (CDA) constitute a group of rare anemias with DAT-negative hemolysis, dysplastic and ineffective erythropoiesis. Three major types, I, II, and III, and several subgroups have been identified [8]. MCF after EMA staining is reduced in CDA II and pyropoikilocytosis $[2,9]$. CD59 also seems to be slightly reduced in pyropoikilocytosis, albeit only studied in a few cases [10].

CDA III is the rarest form of CDA. At least two forms of CDA III exist, one familial which is inherited in an autosomal dominant manner, and one sporadic that might be inherited as an autosomal recessive trait or arising as de novo spontaneous mutation [11-13]. The largest known family with CDA III originates from the Swedish county of Vasterbotten [11]. The detection of the mutated gene linked to CDA I, Codanin-1, and the mutated gene SEC23B in CDA II, has facilitated the diagnosis in these two subtypes of CDA [14-16]. We have recently found that a mutation in KIF23 is associated with CDA III in two unrelated families, so a genetic diagnostic approach is now available for the three major types of CDA [17].
We have previously studied the erythrocyte membrane in two patients with CDA III [18]. The study, which was based on monoclonal antibodies and gel electrophoresis, did not reveal any gross alteration of the erythrocyte membrane concerning CD44, CD47, CD59 and Rh-related proteins, but there was a slight reduction of glycosylation of band 3. The osmotic fragility test appeared normal in 10 CDA III patients from the Västerbotten family [11].

The aim of this study was to examine the erythrocyte membrane in CDA III with flow cytometry using EMA-binding test and monoclonal antibodies against CD55 and CD59. These assays are routinely performed in the diagnostic workup of DAT-negative hemolytic anemia. It is unknown if flow cytometry with EMA and monoclonal antibodies against CD55 and CD59 can be used to identify samples from CDA III patients.

The study was approved by the Regional Ethical Review Board in Umea, Sweden, dnr: 2010-8-31.

\section{Materials and Methods}

To our knowledge, there are 59 known persons alive with CDA III or with one parent diagnosed with CDA III, in the Västerbotten family. Seven affected and one unaffected person, living abroad, was not contacted for the study. Because of mental retardation, two adults

*Corresponding author: Maria Liljeholm, Department of Radiation Sciences, Umea University, SE-90185 Umea, Sweden, E-mail: maria.Iiljeholm@vll.se

Received September 24, 2013; Accepted November 06, 2013; Published November 10, 2013

Citation: Liljeholm M, Gronlund E, Golovleva I, Sandström H, Wahlin A (2013) Erythrocyte Flow Cytometric Analysis in Congenital Dyserythropoietic Anemia Type III-Evaluation of Eosin-5'-Maleimide, CD55, and CD59. J Blood Disorders Transf 4: 172. doi: 10.4172/2155-9864.1000172

Copyright: (c) 2013 Liljeholm M, et al. This is an open-access article distributed under the terms of the Creative Commons Attribution License, which permits unrestricted use, distribution, and reproduction in any medium, provided the original author and source are credited. 
with unknown CDA-status were also excluded. The remaining 49 family members were contacted and informed of the study by mail. A few days after mail correspondence the family members were contacted by phone and asked if they wanted to participate. Concerning children under the age of 18 , their parents were contacted. Children between 15 to 18 years of age were given information specially designed for this age group. 30 out of 43 adults but none of the 6 children wanted to participate. The p.P916R mutation of KIF23 was confirmed in all CDA III positive cases and absent in all CDA III negative cases. Study sample characteristics are shown in (Table 1).

Ten ml peripheral blood, divided into 2 tubes, was obtained at the nearest hospital or in the nearest district health care centre. One sample was sent to the nearest hospital to be analysed the same day concerning hemoglobin $(\mathrm{Hb})$ and erythrocyte cellular volume (MCV). The other tube was sent to the Hematopathology Laboratory in Umeå for flow cytometric assays. Flow cytometry was performed within 24 hours.

\section{Flow cytometry}

Flow cytometric assays were performed on a BD Biosciences FACS Calibur flow cytometer. Mean channel fluorescence (MCF) levels were determined by Cellquest software. All assays were performed by one and the same biomedical scientist.

EMA-labelling of red cells and flow cytometry was performed as previously described by King et al. [3]. 16 CDA III positive and 14 CDA III negative individuals were analyzed. In each assay, the study sample from a subject of the Västerbotten family was set up with three normal controls, randomly selected from the hospital routine laboratory, provided that they had hemoglobin, MCV, leukocytes, and platelets within the normal range for our laboratory. Fluorescence intensity in MCF units was determined for 30000 events. Mean-MCF was determined for the study sample as for the three normal controls.

The expression of CD55 and CD59 on erythrocytes was analysed in 12 CDA III positive subjects and 7 CDA III negative siblings. Erythrocytes were prepared and analysed for CD55 and CD59 as described by Sutherland et al. [19]. In each assay the study sample was set up with one normal control. 15000 events were recorded. A threshold for populations of erythrocytes expressing CD55 and CD59 in the normal controls was identified and set at the same level in the study sample.

\section{Statistical analysis}

Ratios of MCF in study samples and control(s) in each assay were calculated. Study sample MCF and control MCF values were normally distributed according to Kolmogorov-Smirnov test. Results are presented as MCF ratios, standard deviation, and range. The Sign test was used to compare MCF of study samples and their normal controls. $\mathrm{p}<0.05$ was regarded as significant. Pearson correlation test was used for analysis of correlation between EMA fluorescence and MCV.

\begin{tabular}{|l|l|l|}
\hline \multicolumn{1}{|c|}{ CDA III positive subjects } & CDA negative relatives \\
\hline No. & 16 & 14 \\
\hline Males/Females & $8 / 8$ & $6 / 8$ \\
\hline Age, years & $55(21-72)$ & $51(27-82)$ \\
\hline Ery-MCV $^{1}(\mathrm{fL})$ & $99(94-109)$ & $88,5(70-94)$ \\
\hline $\mathrm{Hb}^{2}(\mathrm{~g} / \mathrm{L})$ & $119(105-157)$ & $145,5(94-150)$ \\
\hline
\end{tabular}

Values are presented as median and range

${ }^{1}$ Erythrocyte mean corpuscular volume

${ }^{2}$ Hemoglobin

Table 1: Study sample characteristics.

\section{Results}

Mean EMA MCF was higher (1.08) in the CDA III positive group than in their normal controls (1.0) but did not differ between CDA III negative siblings (0.99) and their normal controls (1.0) (Table 2 and Figure 1). The slightly increased EMA fluorescence in CDA III patients was significant $(\mathrm{p}<0.001)$. There was a significant correlation between EMA-MCF and MCV ( $\mathrm{r}=0.72, \mathrm{p}=0.01$ ) (Figure 2). The expression of CD55 and CD59 did not differ between the CDA III positive patients and their controls ( 0.99 and 1.01, respectively), nor between the CDA III negative siblings and their normal controls ( 1.04 and 0.99 , respectively) (Table 2 and Figure 3). Correlation analysis between MCV and CD55 or CD59 could not be performed due to the small study sample.

\begin{tabular}{|l|c|cc|c|c|}
\hline & $\mathbf{n}$ & \multicolumn{2}{|c|}{$\begin{array}{c}\text { CDA III pos./controls } \\
\text { ratios and (range) }\end{array}$} & $\mathbf{n}$ & $\begin{array}{c}\text { CDA III neg. relatives/ } \\
\text { controls } \\
\text { ratios and (range) }\end{array}$ \\
\hline EMA MCF & 16 & $1.08^{* * *}(1.01-1.17)$ & 14 & $0.99(0.89-1.08)$ \\
\hline CD55 & 11 & 0.99 & $(0.96-1.00)$ & 7 & $1.04(0.99-1.32)$ \\
\hline CD59 & 11 & 1.01 & $(0.96-1.15)$ & 7 & $0.99(0.97-1.02)$ \\
\hline
\end{tabular}

*** $p<0.001$

Table 2: Summary of flow cytometry results.

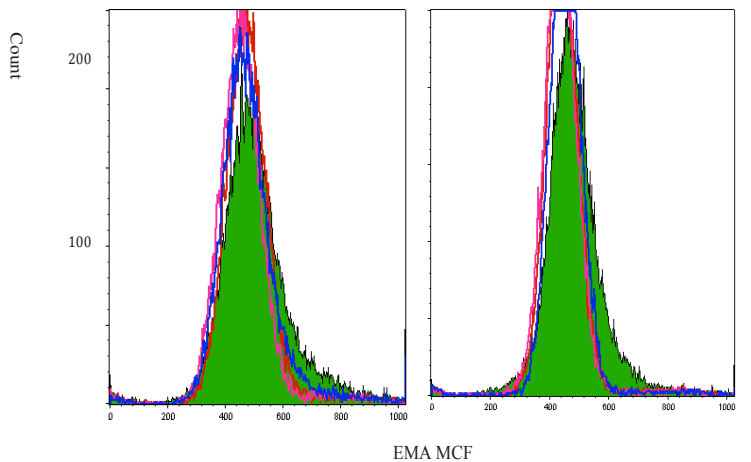

Figure 1: Fluorescence histograms for two patients with CDA III. Three normal controls in each assay. Erythrocytes incubated with EMA. Green areas constitute the CDA III patients. Flourescence is slightly increased in CDA III compared to normal controls.

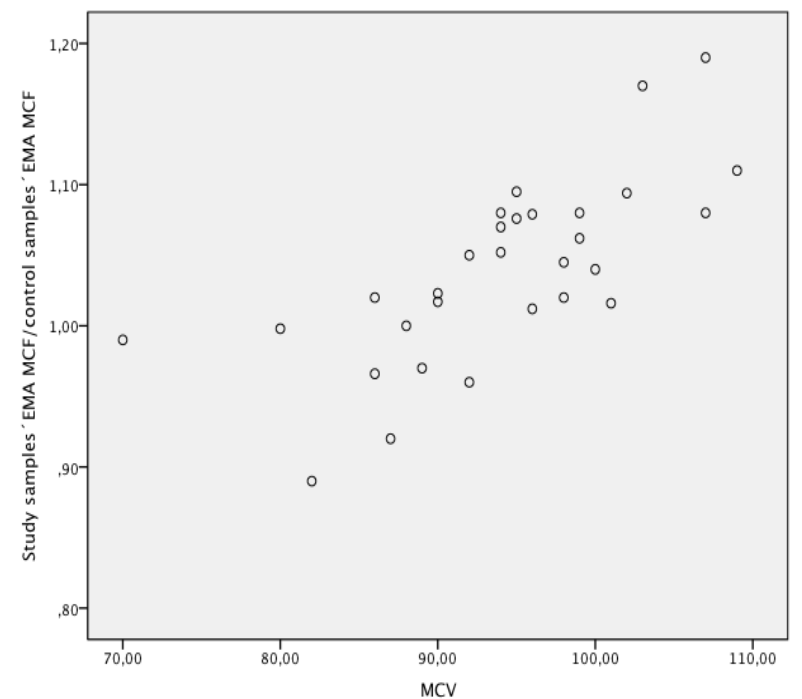

Figure 2: Positive correlation between MCV and EMA MCF was established, $r=0.715, p=0.01$. 
Citation: Liljeholm M, Gronlund E, Golovleva I, Sandström H, Wahlin A(2013) Erythrocyte Flow Cytometric Analysis in Congenital Dyserythropoietic Anemia Type III-Evaluation of Eosin-5'-Maleimide, CD55, and CD59. J Blood Disorders Transf 4: 172. doi: 10.4172/2155-9864.1000172

Page 3 of 3

\section{Discussion}

Reduced fluorescence intensity of intact red cells after incubation with EMA has been reported in patients with CDA II by King et al., and we have previously found indications of an abnormality in band 3 in patients with CDA III $[3,18]$. In the present study, we performed flow cytometry of erythrocytes from members of a family with CDA III. Unexpectedly, we found that the fluorescence intensity of red blood cells after EMA incubation is higher in samples from patients with CDA III than in normal controls, but this difference was correlated to higher $\mathrm{MCV}$ in the patients compared to the normal controls. This finding motivated us to perform an analysis of EMA fluorescence and MCV in 22 random hospital patient samples that were analysed by us at the same time period. We found a significant $(r=0.5, p=0.02)$ correlation between EMA fluorescence and MCV also in this cohort.

EMA MCF was normal in samples from normal siblings of the CDA III patients.

The flow cytometric profiles of CD55 and CD59 did not differ between patients with CDA III and normal controls.

The affinity of EMA to the membrane protein band 3 is high, which is the main cause of lower fluorescence in hereditary spherocytosis. To a minor extent EMA also interacts with CD47 and Rh-antigen, also reduced in hereditary spherocytosis, contributing to the reduced fluorescence after EMA staining in this disease [4]. The reduced fluorescence in CDA II is probably not due to a quantitative reduction of band 3 in the erythrocyte membrane but rather an affected configuration leading to a decreased affinity to EMA [6]. The increase in EMA fluorescence in CDA III is probably due to the slight increase of erythrocyte volume (MCV) exposing a larger surface per cell to binding of EMA to band 3, Rhesus-antigen and CD47 on the erythrocyte membrane. A correlation has been confirmed in individuals with high MCV due to overconsumption of alcohol, but in DAT-positive hemolytic anemia no correlation between MCV and MCF was observed [3]. Low MCV due to iron deficiency does not affect EMA MCF $[3,6,20]$. The results of the present study indicate no abnormality of the erythrocyte membrane in CDA III and show that standard flow cytometry cannot be used to discriminate between CDA III and normal controls.

\section{Acknowledgements}

We thank all members of the CDA III family in Västerbotten and acknowledge collaboration with Göran Roos, Professor at the Department of Medical Biosciences, Umeå University and Hematopathology Laboratory at Umeå University hospital.

Financial support was obtained through regional agreement between Umeå University and Västerbotten County Council on cooperation in the field of Medicine Odontology and Health (ALF), and unrestricted grants from Cancerforskningsfonden Norrland.

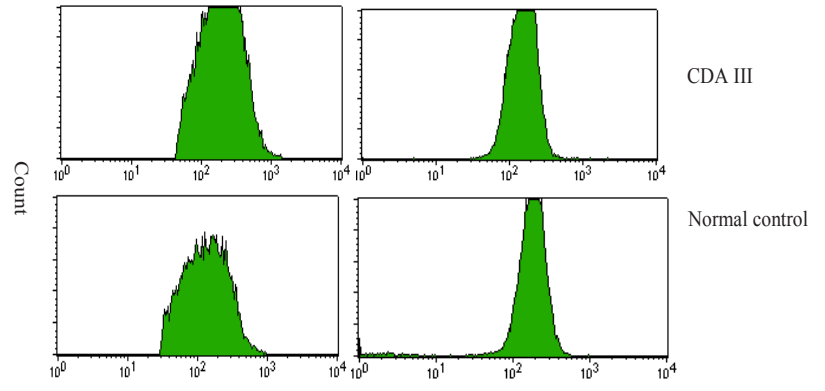

Figure 3: Similar expression of CD 55 and CD 59 in one patient with CDA III and one normal control.

\section{Authorship}

Contribution: M.L., A.W., and E.G. designed the study; M.L. collected clinical data and samples from the CDA III family; E.G. collected control samples and performed flow cytometry analyses. M.L., and A.W. analyzed an interpreted flow cytometry data. M.L. and A.W. wrote the paper and all authors contributed to the writing process and approved the final manuscript.

\section{References}

1. Bianchi P, Fermo E, Vercellati C, Marcello AP, Porretti L, et al. (2012) Diagnostic power of laboratory tests for hereditary spherocytosis: a comparison study in 150 patients grouped according to molecular and clinical characteristics. Haematologica 97: 516-523.

2. King MJ, Zanella A (2013) Hereditary red cell membrane disorders and laboratory diagnostic testing. Int J Lab Hematol 35: 237-243.

3. King MJ, Behrens J, Rogers C, Flynn C, Greenwood D, et al. (2000) Rapid flow cytometric test for the diagnosis of membrane cytoskeleton-associated haemolytic anaemia. Br J Haematol 111: 924-933.

4. King MJ, Smythe JS, Mushens R (2004) Eosin-5-maleimide binding to band 3 and Rh-related proteins forms the basis of a screening test for hereditary spherocytosis. Br J Haematol 124: 106-113.

5. Kar R, Mishra P, Pati HP (2010) Evaluation of eosin-5-maleimide flow cytometric test in diagnosis of hereditary spherocytosis. Int J Lab Hematol 32: 8-16.

6. Kedar PS, Colah RB, Kulkarni S, Ghosh K, Mohanty D (2003) Experience with eosin-5'-maleimide as a diagnostic tool for red cell membrane cytoskeleton disorders. Clin Lab Haematol 25: 373-376.

7. Richards SJ, Rawstron AC, Hillmen P (2000) Application of flow cytometry to the diagnosis of paroxysmal nocturnal hemoglobinuria. Cytometry 42: 223-233.

8. Iolascon A, Esposito MR, Russo R (2012) Clinical aspects and pathogenesis of congenital dyserythropoietic anemias: from morphology to molecular approach. Haematologica 97: 1786-1794.

9. King MJ, Telfer P, MacKinnon H, Langabeer L, McMahon C, et al. (2008) Using the eosin-5-maleimide binding test in the differential diagnosis of hereditary spherocytosis and hereditary pyropoikilocytosis. Cytometry B Clin Cytom 74 244-250.

10. King MJ, Jepson MA, Guest A, Mushens R (2011) Detection of hereditary pyropoikilocytosis by the eosin-5-maleimide (EMA)-binding test is attributable to a marked reduction in EMA-reactive transmembrane proteins. Int $\mathrm{J}$ Lab Hematol 33: 205-211.

11. Bergstrom I, Jacobsson L (1962) Hereditary benign erythroreticulosis. Blood 19: $296-303$

12. Goudsmit R, Beckers D, De Bruijne JI, Engelfriet CP, James J, et al. (1972) Congenital dyserythropoietic anaemia, type 3. Br J Haematol 23: 97-105.

13. Wickramasinghe SN (1998) Congenital dyserythropoietic anaemias: clinica features, haematological morphology and new biochemical data. Blood Rev 12: $178-200$.

14. Dgany O, Avidan N, Delaunay J, Krasnov T, Shalmon L, et al. (2002) Congenital dyserythropoietic anemia type I is caused by mutations in codanin-1. Am J Hum Genet 71: 1467-1474.

5. Bianchi P, Fermo E, Vercellati C, Boschetti C, Barcellini W, et al. (2009) Congenital dyserythropoietic anemia type II (CDAll) is caused by mutations in the SEC23B gene. Hum Mutat 30: 1292-1298

16. Schwarz K, lolascon A, Verissimo F, Trede NS, Horsley W, et al. (2009) Mutations affecting the secretory COPII coat component SEC23B cause congenital dyserythropoietic anemia type II. Nat Genet 41: 936-940.

17. Liljeholm M, Irvine AF, Vikberg AL, Norberg A, Month S, et al. (2013) Congenita dyserythropoietic anemia type III (CDA III) is caused by a mutation in kinesin family member, KIF23. Blood 121: 4791-4799.

18. Wickramasinghe SN, Wahlin A, Anstee D, Parsons SF, Stopps G, et al (1993) Observations on two members of the Swedish family with congenita dyserythropoietic anaemia, type III. Eur J Haematol 50: 213-221.

19. Sutherland DR, Kuek N, Davidson J, Barth D, Chang H, et al. (2007) Diagnosing PNH with FLAER and multiparameter flow cytometry. Cytometry B Clin Cytom 72: 167-177

20. D'Alcamo E, Agrigento V, Sclafani S, Vitrano A, Cuccia L, et al. (2011) Reliability of EMA binding test in the diagnosis of hereditary spherocytosis in Italian patients. Acta Haematol 125: 136-140. 